\title{
ON INEQUALITIES FOR QUERMASSINTEGRALS AND DUAL QUERMASSINTEGRALS OF DIFFERENCE BODIES
}

\author{
WEIDONG WANG
}

Abstract. In this paper, inequalities for quermassintegrals and dual quermassintegrals of difference bodies are given. In particular, an extension of the Rogers-Shephard inequality is obtained.

Mathematics subject classification (2010): 52A40, 52A20.

Keywords and phrases: quermassintegrals, dual quermassintegrals, difference body, Rogers-Shephard inequality, extension.

\section{REFERENCES}

[1] G. D. Chakerian, Inequalities for the difference body of a convex body, Proc. Amer. Math. Soc. 18 (1967), 879-884.

[2] R. J. Gardner, Geometric Tomography, Cambridge Univ. Press, Cambridge, UK, 2nd edition, 2006.

[3] R. J. Gardner And G. Y. Zhang, Affine inequalities and radial mean bodies, Amer. J. Math. 120 (1998), 505-528.

[4] G. H. Hardy, J. E. Littlewood and G. Pólya, Inequalities, Cambridge University Press, Cambridge, 1959.

[5] E. Lutwak, Dual mixed volumes, Pacific J. Math. 58 (1975), 531-538.

[6] C. A. Rogers AND G. C. Shephard, The difference body of a convex body, Arch. Math. 8 (1957), 220-233.

[7] R. SCHNEIDER, Eine verallgemeinerung des differenzenkörpers, Monatsh. Math. 74 (1970), 258-272.

[8] R. SchneIder, Convex Bodies: The Brunn-Minkowski theory, Cambridge Univ. Press, Cambridge, 1993. 